\title{
An aggregate approach to simulate evacuation of a building
}

\author{
M. Di Gangi ${ }^{1}$ \& C. Rindone ${ }^{2}$ \\ ${ }^{I}$ Department of Civil Engineering, University of Messina, Italy \\ ${ }^{2}$ Department of Computer Science, Mathematics, Electronics and \\ Transportation, Mediterranea University of Reggio Calabria, Italy
}

\begin{abstract}
This paper concerns a method for the simulation of pedestrian outflow related to the evacuation of a building. In particular the proposed method allows an aggregate estimation of evacuation time of a building. The method can be easily implemented and can be used to give a first evaluation of evacuation procedures without performing evacuation drills and can be used to give a quickly response in identifying critical points on the network. To check the capabilities of the proposed approach a comparison between results obtained from simulations and data recorded from an experiment on a test site conducted in a primary school located in an Italian town. The adopted methodology can be applied to any building with homogeneous characteristics in terms of activities (i.e. offices, banks, commercials).

Experimentation was carried out by the Laboratory for Transport Systems Analysis (LAST) of the University Mediterranea of Reggio Calabria (Italy) under a research project entitled SURE, whose general objective was risk reduction in urban areas in terms of exposure through the definition and implementation of evacuation procedures.
\end{abstract}

\section{Introduction}

One of the aspects that have in the last years attracted the attention of some experts of the field on transport model consists of the analysis of working modality of a transport system when it is subjected to emergency conditions. That means to analyze how to reformulate some approaches commonly used in ordinary conditions in order to take into account of the particular operative 
conditions due to the emergency status of the transport system. Such approach should allow the availability of tools able to conduct simulations of evacuations avoiding expensive drills or to give useful indications to enhance evacuation processes of different extended areas (building, industrial site, town, etc.).

Traditionally risk is defined as the product of three factors: probability, vulnerability and exposure (of a population, goods, etc.) [1]. In order to reduce risk, attention have been usually turned to two main components as the probability that the event occurs (whenever possible, that is essentially for anthropic events) and the vulnerability related to resistance of infrastructures.

To dishearten risk it is also possible to operate on exposure component if, since the occurring of the event to the propagation of the effects to population, it is possible to evacuate the whole (or a part of the) population.

Effects produced in the space connected to disaster event can be: punctual [2]; of area or diffused [3]. These effects change on time with temporal evolution of event. For same kind of disasters (i.e. tsunami, presence of a bomb, etc...), during time interval it is possible to intervene to reduce risk. On the contrary, for other kind of events (i.e. earthquakes), the duration of this time interval is very short. Within this work only events with delayed effects have been taken into consideration. In this paper we focus on punctual delayed effects on time [3].

An evacuation can be defined as a general mobilization of people (and/or goods) due to the occurrence of a calamitous event. Its main objective is to reduce the number of people (and/or goods) present in the area where the event strikes [4]. Evacuation drills are mainly performed to practice the people to leave the interested area; these tests can also be used for getting information concerning the behavior of the people in order to build a set of mathematical models able to reproduce the evacuation. These models can constitute a Decision Support System (DSS) to be used for planning emergences [5].

Simulation models, used to perform quantitative analysis on the operational conditions of a transportation system in emergency circumstances, differ depending on the hypotheses made on the representation of flow characteristics.

Many studies (i.e. [6-8]) highlighted the limits of static assignment models in analyzing phenomena connected to temporal variations in terms of both demand and supply, such as rising and scattering of queues due to temporary peaks of demand and/or capacity reductions of infrastructures. Thus, in order to comply with these phenomena, it is necessary either to use static models in pseudodynamic assignment procedures [9], or to remove the intra-period stationarity hypothesis and choose within-day dynamic assignment models (Dynamic Traffic Assignment - DTA).

In general, a classification of DTA models can be made according to the representation of traffic variables (continuous or discrete) and on the aspect of the variables representing network performances (aggregate or disaggregate). Socalled macroscopic models, or flow-based analytical models [10-14], simulate network performances by means of aggregate variables (speed, density, flow) with explicit capacity, as in static models, and use a continuous representation of traffic; generally, in formulating macroscopic models, fluid-dynamic analogies of traffic are adopted. A second type of model, named mesoscopic [15-21], is similar to the 
previous one in the way it simulates network performances (aggregate variables with explicit capacity are used), but it differs in terms of traffic representation; the peculiarity of mesoscopic models consists of a discrete flow representation for groups of vehicles/users. A third type is made up by microscopic models; where individual trajectories of all vehicles are simulated by using disaggregate variables with implicit capacity, and a discrete traffic representation.

Computational complexity of cited models requires us to analyze outflow conditions of networks, the adoption of dedicated and sophisticated software. On the other hand there is also, in certain circumstances, a need for simple tools able to quantify, in terms of evacuation time, the effectiveness of an evacuation plan.

In this paper a specific methodology to simulate operations of a transportation system in emergency conditions is shown. In particular it is presented a method (model and procedure) to simulate evacuation of buildings. An aggregate approach (macroscopic) is proposed; it allows a prompt first attempt application in case of the lack of commercial software or tools suitable to perform micro simulations.

The paper is structured as follows: in section 2 it is described an aggregate method to compute evacuation time in case of buildings, starting from the procedure proposed for ships by the International Maritime Organization (IMO) (Here are reported those parts of the procedure required in the case of a building; refer to the original document for the integral version used for the evacuation of ships.) [22]; section 3 reports an application of the proposed method and procedures adopted to carry out simulations in a real context. Section 4 presents an analysis of results obtained with the simulations and a comparison between these results and on site experimentation data.

\section{An aggregate method to compute the evacuation time}

The proposal of this method arises from the need to have a tool, even though approximate, able to give some indications both on the outflow conditions and on the evacuation times of a building.

The proposed macroscopic approach applies to a building an adaptation of the International Maritime Organization guidelines [22] on evacuation analysis for passenger ships. Concerning the adaptation of such methodology to the context of a building, it is worth noting that both data and parameters considered in the considered IMO guidelines are based on data experienced on civil residences.

One of the advantages of this method consists of the possibility to evaluate evacuation times with the only support of a common worksheet application, avoiding the necessity to use specific commercial software.

\subsection{Main hypotheses}

The proposed method of estimating evacuation time is based on the following assumptions:

- all occupants will begin evacuation at the same time and will not hinder each other;

- occupants will evacuate via the main escape route; 
- initial walking speed depends on the density of persons, assuming that the flow is only in the direction of the escape route, and that there is no overtaking;

- full availability of escape arrangements is considered, unless otherwise stated;

- people can move unhindered;

- contra flow is accounted for by a contra flow correction factor;

- effects of passenger age and mobility impairment, flexibility of arrangements, unavailability of corridors, restricted visibility due to smoke, are accounted for in a correction factor and a safety factor.

Different scenarios should be considered for the analysis of evacuation considering different numbers and distributions of occupants.

The network is represented using fundamentals of graph theory. The classes of components making up the graphs are:

- area centroids: these nodes represent the barycentre of each area (rooms, offices, etc.) comprising the building. They sum up the origins of the trips of all the people who, at emergency time, are within the area considered. There is a centroid for each area in which the building is divided;

- destination nodes (centroids): these represent the safe areas towards which people converge in an emergency. Thus they correspond to the destination of each path;

- $\quad$ network nodes: these are located at each potential change of direction along a generic path or at significant variations in geometric and/or functional characteristics of a trunk(i.e. width variations);

- real arcs: these represent the connection between two network nodes or a network and a destination node; they coincide with trunks of the pedestrian network and are classified into flat ramps (corridors) and descending ramps (stairs) or with trunks or road network where the collecting bus moves;

- connector arcs: these represent the connection between a centroid and a network node.

\subsection{Methodology}

Proposed method can be summarized through a succession of operations finalized to the evaluation of evacuation time. Such operations are listed in the following and are depicted in the diagram shown in Fig. 1.

\subsubsection{Data analysis}

- Schematization of escape route and data acquisition. In this step a scheme of escape route is built up considering geometric characteristics of elements of the building. Data concerning occupants defining both their number and their positions are also acquired.

\subsubsection{Supply model}

- Graph assembly. In this step the network of all possible pathways is represented by means of a graph considering the elements (corridors, stairs, etc.) that they are composed of. 
- Definition of escape route. In this step, destination assembly point and paths are defined for each area assumed as origin of evacuation; paths are described as a sequence of elements of the graph obtained in the previous step.

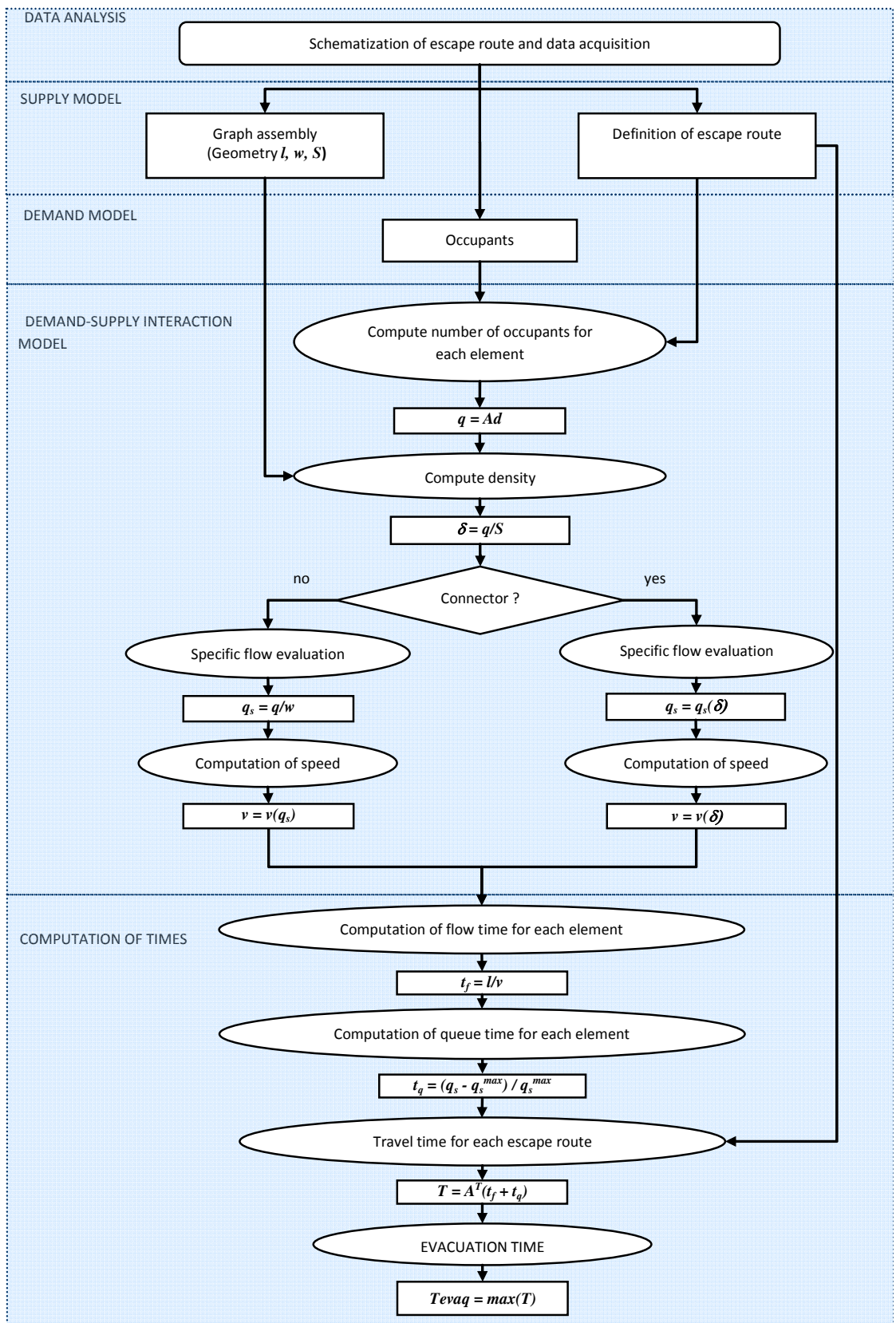

Figure 1: Scheme of the operations performed during simulation. 


\subsubsection{Demand model}

- Computation of occupants. Starting from data concerning occupants (number and positions), the number $d_{i}$ of occupants of a place is attributed to each correspondent origin node, obtaining the demand vector $\boldsymbol{d}$.

\subsubsection{Demand-supply interaction model}

- Computation of occupants for each element $q_{i}$. For each element of escape routes, the flow of occupants is computed using the arc-path incidence matrix (A) as $\boldsymbol{q}=\boldsymbol{A d}$.

- Computation of the specific flow $\left(q_{s}\right)$. This value is computed dividing flow $q$ by the effective width $w$ of the considered element except for connectors; for those latter components specific relationships based on density are adopted.

- Computation of speed. Once specific flow $q_{s}$ is known, the following two cases arise:

- values of $q_{s}$ do not reach the value $q_{s}^{\max }$ of characteristic maximum specific flow for the considered element; speed $v$ ' is computed using specific relationships depending on specific flow;

- values of $q_{s}$ overtake the value $q_{s}{ }^{\max }$ of characteristic maximum specific flow for the considered element; in this case queues arise in correspondence of transition points; correspondent speed $v$ ' ' is given by the limit value indicated by relationships expressing speeds depending on specific flow.

\subsubsection{Computation of times}

- Computation of flow time for each element $\left(t f_{i}\right)$. Those values are computed once evaluated walking speed for each considered element as $t f_{i}=l_{i} / v_{i}$.

- Computation of queue time for each element $\left(t q_{i}\right)$. Those values are computed depending on specific flow $q_{s}$ and the value $q_{s}{ }^{\max }$ of characteristic maximum specific flow for the considered element as $t q_{i}=\left(q_{s}-q_{s}^{\max }\right) / q_{s}^{\max }$.

- Computation of travel time for each escape route (T). Travel times are computed, for the whole set of evacuation routes, considering the arc-path incidence matrix as $\boldsymbol{T}=\boldsymbol{A}^{T}\left(\boldsymbol{t}_{\boldsymbol{f}}+\boldsymbol{t}_{q}\right)$. This procedure is repeated for each scenario (i.e. daytime and night time).

- Computation of evacuation time. After computing travel time for each evacuation route, the higher value $T$ among all the travel times is considered as the evacuation time.

\section{Applicative context}

\subsection{The test site}

The Methodology is applied to verify the school evacuation plan in a town (Melito Porto Salvo) of south Italy. The scenario simulated concerns an incident involving a tank transporting hazardous goods which, on a workday morning $(8.00 \mathrm{am}-12.00)$, is supposed to leak. In the instant $t 1$, the presence of a 
potentially disastrous event is announced. The mayor decides that the surrounding area, that contains the school building, must be evacuated [23].

The school evacuation plan stipulates that everybody must gather at a site in front of the building (called first assembly point); according to the town evacuation plan, the school's staff and pupils will be led to the refuge area located about $2 \mathrm{~km}$ from the school by means of a bus service starting from another gathering place (second assembly point) as shown in Fig. 2.

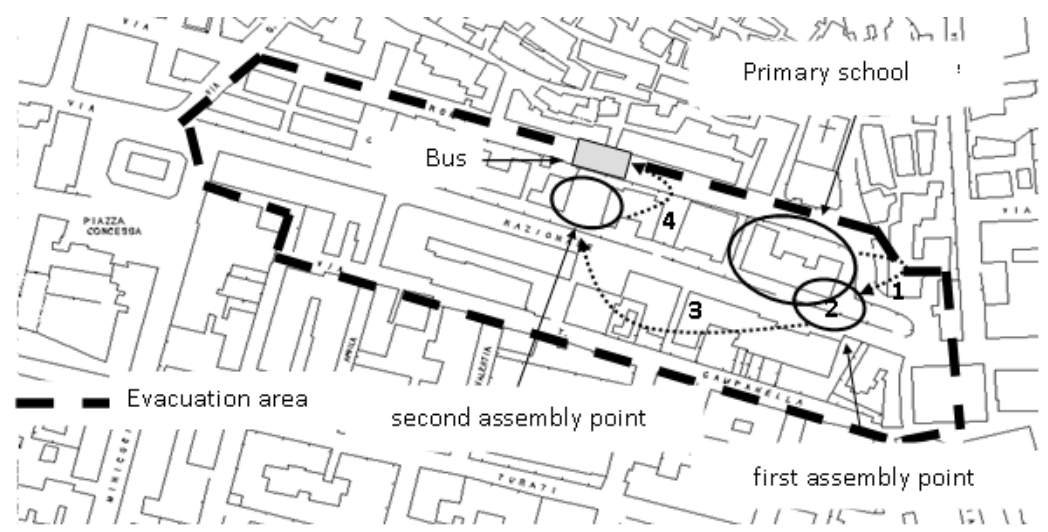

Figure 2: $\quad$ Phases of evacuation.

\subsection{Monitoring}

Two evacuation tests (drills) are carried out: in the first test the town hall and school buildings are involved; in the second test, besides public buildings, private and commercial buildings in the area are involved. To compare the results of two drills, the measured evacuation times of school will be reported below.

Hence evacuation of the school was schematized in the following five main phases: 1) evacuation of the building reaching first assembly point; 2) roll-call of pupils at first assembly point; 3) transfer to second assembly point; 4) boarding on bus; 5) transfer to refuge area. In the application here described the analysis focuses on the evacuation of the first three phases.

Data were gathered concerning supply and demand. During the drill a monitoring system was arranged, with manual/automatic tools and 12 video cameras, in order to acquire data concerning pedestrian outflow (times, densities) both inside and outside the building until the gathering places were reached.

\subsection{Methodology application}

The application has been performed applying to the building an adaptation of the IMO guidelines [22] on evacuation analysis for passenger ships proposed in [2]. This application can be summarized as follows:

- Schematization of escape routes as a hydraulic network where the pipes are the corridors and stairways, the valves are the doors and restriction in general and the tank are the public spaces. 
- Calculation of the density $\delta$ for all the escape routes.

- Calculation of the initial specific flow $q_{s}^{0}$, as a function of the densities.

- Calculation of the flow $q$ for corridors and doors, in the direction of the assigned escape routes.

- Calculation of the outlet calculated flows $q$ for each transition point.

- Calculation, from the values of $M$ (number of persons entering a flight or a corridor) and of $q$, of the flow time $T_{F}$ for each stairway and corridor.

- Calculation of travel time $T_{D}$ from the farthest point of escape route to the stairway.

- Calculation, for each stair flight, of its travel time $T_{S}$. For each floor the total stair travel time is given by the sum of the travel times of all stairs flight connecting the floor with the assembly point.

- Calculation of travel time from the end of the stairway to the assembly point.

- Calculation of the overall time $T_{I}$ to travel along an escape route to the assembly point.

- Once the calculation is performed for all the escape routes, the highest $T_{I}$ is selected for calculating the travel time as $T_{T}=\left(f_{1}+f_{2}\right) T_{I}$ where $f_{l}$ and $f_{2}$ are correction factors used to take into account of conditions of the simulated scenario and of contra flow.

\subsection{Mesoscopic simulation}

To validate results of methodology, a mesoscopic simulation is carried out. Simulation is realised applying a Dynamic Traffic Assignment (DTA) model [24-27]. Applying this model, a simulation of the observed evacuation was performed. Paths were obtained from the school evacuation plan. As regards the cost functions adopted, for fictitious links a constant speed function was considered; for corridors and descending flights relationships between speed and specific flow specified and calibrated in [28] has been considered.

Demand values used in the simulation were obtained from school attendance on the experimentation day, and users were located in offices and classrooms following the real distribution. The demand value to be evacuated consists of about 150 users and paths were obtained directly from the school evacuation plan. The first three steps previously identified were simulated with a dynamic approach.

\subsection{Comparison of results}

Results obtained from the application of the above-described approaches have been expressed in terms of evacuation time. In Tab. 1 evacuation times for the considered phases related to experimentation and to simulations are reported.

Some consideration can be made on the proposed approaches. For the mesoscopic simulation the main advantage consists of the possibility to explicitly simulate queues and spill backs, whilst a drawback is given from the necessity to use a specific piece of software. The proposed methodology gives an aggregate representation of flow conditions and does not allow a detailed 
analysis of them; on the other hand it can be easily implemented on a spreadsheet.

Table 1: Comparison of evacuation time obtained from drill with simulated ones.

\begin{tabular}{lccc}
\hline \multicolumn{1}{c}{ Phase } & $\begin{array}{c}\text { Measured } \\
\text { time }\end{array}$ & $\begin{array}{c}\text { Proposed } \\
\text { method }\end{array}$ & Mesosimulation \\
\hline 1) evacuation of building reaching first assembly point & $4^{\prime} 14^{\prime \prime}$ & $4^{\prime} 23^{\prime \prime}$ & $5^{\prime} 47^{\prime \prime}$ \\
2) roll-call of pupils at first assembly point & $3^{\prime} 05^{\prime \prime}$ & $3^{\prime} 00^{\prime \prime}$ & $2^{\prime} 58^{\prime \prime}$ \\
3) transfer to second assembly point & $2^{\prime} 00^{\prime \prime}$ & $4^{\prime} 00^{\prime \prime}$ & $1^{\prime} 43^{\prime \prime}$ \\
\hline Total time & $9^{\prime} 19^{\prime \prime}$ & $11^{\prime} 23^{\prime \prime}$ & $10^{\prime} 28^{\prime \prime}$ \\
\hline
\end{tabular}

\section{Conclusions and perspectives}

The main result of this paper concerns both the application of a mesoscopic dynamic network assignment model in a multimodal context and the specification and calibration of some cost functions adopted in this model. A comparison between experimental data and simulation results shows how the usage of appropriate simulation models can realistically reproduce user behavior. It was shown that such models could be used as a support both to verify effectiveness of existing evacuation plans without resorting to expensive drills and to draw up evacuation plans. Implementation of appropriate cost functions can make the applied methodologies suitable for any building and/or area with homogeneous characteristics in terms of activities. Further investigations on travel time functions under different operative conditions are under development.

\section{References}

[1] Russo, F., Vitetta, A., Risk evaluation in a transportation system. International Journal of Sustainable Development and Planning, 1 (2), pp. 170-191, 2006.

[2] Di Gangi, M., Velonà, P., Deflusso pedonale negli edifici in condizioni di emergenza - modelli e metodi per la simulazione, applicazione ad un caso reale. Franco Angeli, Milano ISBN 978-884649-065-0, 2007.

[3] Russo, F., Chilà G., Safety of users in road evacuation: demand model. In Urban Transport XIII, Brebbia C.A. (ed.), WIT Press pp. 773-782 ISBN 978-184564-087-3, 2007.

[4] Russo F., Chilà G., A sequential dynamic choice model to simulate demand in evacuation conditions. Proc. of Risk analysis VII \& Brownfields V, Brebbia C. A. (ed.), WIT Press, Southampton, pp. 431-442, 2010.

[5] Russo F., Rindone C., Evaluation methods for evacuation planning. Proc. of Urban Transport XVI, Urban Transport and the Environment in the 21st century, Brebbia C. A. (ed.), WIT Press, Southampton, pp. 335-343, 2010.

[6] Ben Akiva, M., Dynamic network equilibrium research. Transportation Research Part A: General Volume 19, Issues 5-6, Pages 429-431, 1985. 
[7] Friesz, T. L., Transportation network equilibrium, design and aggregation: key development and research opportunities. Transportation Research Part A: General, Volume 19, Issues 5-6, Pages 413-427, 1985.

[8] Cascetta, E., Transportation systems engineering: theory and methods, Kluwer, 2001.

[9] Russo, F., Vitetta, A., Urban road transportation analysis in emergency conditions: models and algorithms. Proceedings of Urban Transport 2000, Wessex Institute of Technology. Sucharov e Brebbia ed., WIT Press Boston, pp. 533-542, 2000.

[10] Merchant, D.K., Nemhauser, G.L., A model and an algorithm for the dynamic traffic assignment problems. Trans. Sci., 12(3), 183-207, 1978.

[11] Ben Akiva, M., De Palma, A., Kanaraglou P., Dynamic Model of Peak Period Traffic Congestion with Elastic Arrival Rates. Trans. Sci. 20 (2), 164-181, 1986.

[12] Janson, B. N., Dynamic traffic assignment for urban road networks. Transportation Research Part B: Methodological, Volume 25, Issues 2-3, April-June 1991, Pages 143-161, 1991.

[13] Carey, M., Nonconvexity of the dynamic assignment problem. Operations Research, 35 No. 5, 58-69, 1992.

[14] Papageorgiou, M., Dynamic modeling, assignment, and route guidance in traffic networks Transportation Research Part B: Methodological, Volume 24, Issue 6, pp. 471-495, 1990.

[15] Smith, M. J., Van Vuren, T. Traffic equilibrium with responsive traffic control. Trans. Sci. Vol. 27, No. 2, pp. 118-132, 1993

[16] Ben Akiva, M., Cascetta, E., Gunn, H., Smulders, S., Whittaker, J., DYNA: a real-time monitoring and prediction system for inter-urban motorways. First World Congress on Applications of Transport Telematics and Intelligent Vehicle-Highway Systems. Paris, 1994.

[17] Ben Akiva, M., Bierlaire, M., Bottom, J., Koutsopoulos, H. N., Mishalani, R., Development of a route guidance generation system for a real-time application.8th IFAC/IFIP/IFORS symposium on transportation systems. Chania, Greece, 1997.

[18] Ben Akiva, M., Bierlaire, M., Koutsopoulos, H. N., Mishalani, R. DynaMIT: a simulation-based system for traffic prediction and guidance generation. DACCORD Short Term Forecasting Workshop TU Delft, 1998.

[19] Cascetta, E., Cantarella, G.E., A Day-to-day and Within-day Dynamic Stochastic Assignment Model. Transpn. Res. 25a (5), 277-291, 1991.

[20] Di Gangi, M., An algorithm for continuous flow Dynamic network loading. VI World Conference on Transportation Research. Lyon, 1992.

[21] Di Gangi, M., Modelling Dynamic Network Loading on Transportation Networks through a Continuous Packet Approach. Mathematical Modelling of Systems, vol. 2, issue 3, pp. 175-196, 1996.

[22] Mahmassani, H. S., Dynamic Network Traffic Assignment and Simulation Methodology for Advanced System Management Applications. Networks and Spatial Economics, Vol. 1:2 (3, pp. 267-292, 2001. 
[23] IMO International Maritime Organization, Guidelines for Evacuation Analysis for New and Existing Passenger Ships MSC.1/Circ.1238, 30 October 2007

[24] Di Gangi M., Musolino G., Rindone C., Vitetta A., An Italian project for exposure reduction in an urban area: experimentation design and DSS development. Proceedings of European Transport Conference. Strasbourg, Sept. 2006, ISBN/ISSN: 1-905701-01-2, 2006.

[25] Di Gangi, M., Russo, F., Vitetta, A., A mesoscopic method for evacuation simulation on passenger ships: models and algorithms. In Pedestrian and Evacuation Dynamics 2003, pp. 197-208 CMS Press, London ISBN 1904521-08-8, 2003.

[26] Di Gangi, M., Approaching the analysis of transport networks in emergency conditions for the design of evacuation plans. In Risk Analysis $I V$, pp. 485494, WIT Press. ISBN 1-85312-736-1, 2004.

[27] Di Gangi M., Planning Evacuation by Means of a Multi-modal Mesoscopic Dynamic Traffic Simulation Model. B. Murgante, G. Borruso, A. Lapucci (Eds.): Geocomputation \& Urban Planning, SCI 176, pp. 99-115. Springer-Verlag Berlin Heidelberg ISBN: 978-3-540-89929-7, 2009.

[28] Di Gangi, M., Modeling Evacuation of a Transport System: Application of a Multimodal Mesoscopic Dynamic Traffic Assignment Model Intelligent Transportation Systems, IEEE Transactions on , vol. PP, no.99, pp.1-10, 0 doi: 10.1109/TITS.2011.2143408 , 2011 URL: http://ieeexplore.ieee.org/ stamp/stamp.jsp?tp=\&arnumber $=5765688 \&$ isnumber $=4358928$

[29] Di Gangi M., Velonà P., Multimodal mesoscopic approach in modelling pedestrian evacuation Transportation Research Record, vol. 2090 / 2009; p. 51-58, ISSN: 0361-1981, doi: 10.3141/2090-06, 2009. 